\title{
Atratividade e qualidade de iscas alimentares na captura da mosca- das-frutas Ceratitis capitata Wiedemann (Diptera: Tephritidae)
}

Attractiveness and quality of food baits in the capture of fruit flies Ceratitis capitata Wiedemann (Diptera: Tephritidae)

Mileny dos Santos de Souza 1
Msc. em Agronomia, Centro de Ciências Agrárias, UFPB Campus Areia.
E-mail: mileny.lopes67@ gmail.com

Robério de Oliveira ${ }^{2}$ Doutor em Agronomia, Centro de Ciências Agrárias, UFPB, Campus Areia E-mail: roberio_b19@yahoo.com.br

Gilmar da Silva Nunes ${ }^{3}$

Msc. em Agronomia, Centro de Ciências Agrárias, UFPB Campus Areia E-mail: gilmarsilvanunes@gmail.com

Mirelly Miguel Porcino ${ }^{4}$ Msc. em Agronomia, Centro de Ciências Agrárias, UFPB Campus Areia E-mail: mirellyagroufpb@gmail.com

Thais Aparecida Vitoriano Dantas 5

Bacharel em Ciências Biológicas, Centro de Ciências Agrárias, UFPB Campus Areia. E-mail: thaisvitorianodantas@gmail.com

Jacinto de Luna Batista
Prof. Associado IV, Dept ${ }^{o}$ de Fitotecnia e Ciências Ambientais, DFCA, Centro de Ciências Agrárias,
UFPB, Campus Areia
E-mail: jacinto@ @cca.ufpb.br

Resumo: As moscas-das-frutas são pragas de grande importância agrícola, por causarem graves prejuízos a fruticultura mundial. Objetivou-se determinar a eficiência e qualidade de atrativos alimentares na captura de Ceratitis capitata. $\mathrm{O}$ experimento foi conduzido em ambiente telado no Laboratório de Entomologia do Centro de Ciências Agrárias da Universidade Federal da Paraíba - CCA/UFPB, Areia-PB. Avaliou-se a eficiência de dois atrativos alimentares comerciais o Bio Anastrepha ${ }^{\circledR}$ e o CeraTrap ${ }^{\circledR}$ nas formas líquida e semissólida. O delineamento experimental usado foi em blocos casualizados em esquema fatorial ( $5 \times 6 \times 2 \times 4)$ sendo: cinco (quatro atrativos + testemunha de água destilada), seis períodos de exposição dos atrativos no ambiente, dois sexos (adultos fêmeas e machos de C. capitata) distribuídos em quatro blocos. Os atrativos foram dispostos no ambiente telado em armadilhas do tipo frasco caça-mosca, onde foram liberados 300 adultos de $C$. capitata a cada período de exposição. Avaliou-se a atratividade ( $\mathrm{n}^{\circ}$ de insetos capturados) e qualidade das iscas alimentares ( $\mathrm{pH}$, proteína bruta \%, conteúdo evaporado). Os atrativos líquidos capturaram mais $C$. capitata do que os semissólidos. A maior atratividade ao longo dos seis períodos de exposição foi do atrativo CeraTrap ${ }^{\circledR}$ liquida e a captura de insetos fêmeas foi maior em relação aos machos. $\mathrm{O}$ atrativo líquido CeraTrap ${ }^{\circledR}$ é o mais indicado para captura desse díptero.

Palavras-chave: Praga quarentenária; Diptera; Iscas alimentares; Manejo integrado de pragas. 
Abstract: Fruit flies are pests of great agricultural importance, as they cause serious damage to the world's fruit-growing. The objective was to determine the efficiency and quality of food attractions in the capture of Ceratitis capitata. The experiment was conducted in an environment of the Entomology Laboratory of the Agricultural Sciences Center of the Federal University of Paraíba - CCA / UFPB, Areia-PB. to study the efficiency of two commercial food attractions Bio Anastrepha ${ }^{\circledR}$ and CeraTrap ${ }^{\circledR}$ in liquid and semi-solid forms. He experimental design was a randomized block design $(5 \times 6 \times 2$ $x$ 4): five (four traits + distilled control water), six periods of exposure of the attractant in the environment, two sexes (adult females and males of $C$. capitata) distributed in four blocks. The attractions were organized in the imprisoned "flask-flask" environment, where 300 adults of $C$. capitata were released each exposure period. The attractiveness (number of insects captured) and quality of food baits $(\mathrm{pH}, \%$ of crude protein, evaporated contents) were evaluated. Liquid attractants captured more C. capitata than the semisolids. The highest attractiveness over the six exposure periods was the attractive CeraTrap $^{\circledR}$ liquid and the capture of female insects was greater in relation to males. The attractive CeraTrap ${ }^{\circledR}$ liquid is the most suitable for capture of this Diptera.

Keywords: Quarantine pest; Diptera; food baits; Integrated pest management.

\section{Introdução}

O Brasil está entre um dos maiores produtores mundiais de frutas, no entanto sua participação nas exportações ainda é pequena. Em parte, devido às exigências fitossanitárias impostas pelos países importadores, em decorrência da presença de pragas quarentenárias a exemplo das moscas-das-frutas pertencentes a diversos gêneros. Um dos principais gêneros é o Ceratitis (PASINI et al., 2015) que apresenta uma única espécie, a Ceratitis capitata, esta que tem grande facilidade adaptativa a climas diversos, ampla variedade de hospedeiros, alta capacidade reprodutiva e uma enorme facilidade de dispersão (ZUCCHI, 2008; ZANARDI et al., 2011).

A fruticultura orgânica ampliou seu mercado consumidor nos últimos anos com o advento de alimentos saudáveis, livres de contaminantes, produzidos dentro de padrões ambientalmente corretos. Neste contexto, os atrativos alimentares utilizados como iscas para captura de insetos adultos ainda se configuram em um dos métodos mais recomendados no monitoramento e controle de diferentes espécies das moscas-das-frutas (NASCIMENTO e CARVALHO, 2000).

O uso de atrativos alimentares como a proteína hidrolisada vegetal na fruticultura brasileira ainda é restrito, podendo se atribuir a isso fatores como a sua disponibilidade no mercado e o seu custo. Dessa forma, os fruticultores dão preferência ao uso de iscas tóxicas e a pulverização por cobertura para o controle desse tefritídeo (LOZANO- 
TOVAR et al., 2015). O menor emprego da proteína hidrolisada vegetal também pode estar relacionado ao seu curto período de atratividade quando exposto em campo, sendo necessário realizar várias trocas durante o ciclo produtivo da cultura, acarretando em maiores custos à produção, não só com a compra do produto, mais também em gastos com a mão-de-obra para manuseio das armadilhas.

Desenvolver atrativos alimentares com um maior período de atratividade e de fácil manuseio ainda é um desafio a ser superado, para assim garantir um monitoramento e controle efetivo desses dípteros, reduzindo também os custos de produção e minimizando os problemas com uso frequente e excessivo de inseticidas químicos. Alguns países da Europa e da América Central já desenvolveram atrativos com um poder de atratividade e de qualidade que podem ser considerados superiores aos hidrolisados proteicos comercializados no Brasil.

A isca líquida CeraTrap ${ }^{\circledR}$ (Bioibérica S.A, Barcelona, Espanha) é constituída de proteínas hidrolisadas (MARÍN, 2010) obtidas da mucosa intestinal do suíno, liberando uma série de compostos voláteis, principalmente aminas e ácidos orgânicos, que tem elevado potencial de atração das fêmeas das moscas-das-frutas e mantem um nível constante de atração que dura por várias semanas (RAMOS et al., 2011, 2012; LASA et al. 2013, 2014; LASA e CRUZ 2014; NAVARRO-LLOPIS e VACAS 2014; PEREACASTELLANOS et al., 2015). Essa isca não contém inseticida em sua composição e seu conteúdo líquido não precisa ser trocado com frequência, sendo apenas reposto o evaporado (PEREA-CASTELLANOS et al., 2015). No entanto, para que o uso desse produto seja difundido no Brasil é necessário desenvolver pesquisas que garantam sua eficiência na captura das espécies e condições climáticas comuns a nossas regiões. Dentro dessa perspectiva objetivou-se determinar a eficiência e qualidade de atrativos alimentares na captura de Ceratitis capitata.

\section{Metodologia}

A criação A pesquisa foi conduzida em ambiente protegido por tela antiafídica $(9 \mathrm{x}$ $6 \mathrm{~m}$ ), no Laboratório de Entomologia do Centro de Ciências Agrárias da Universidade Federal da Paraíba - CCA/UFPB, Areia-PB. Os adultos de Ceratitis capitata utilizados na pesquisa foram oriundos de uma criação massal mantida no laboratório criados em dieta artificial segundo a metodologia descrita por Brito (2007), à temperatura de $25 \pm$ $1{ }^{\circ} \mathrm{C}$, umidade relativa de $70 \pm 10 \%$ e fotofase de 12 horas. 
Foram utilizados dois atrativos alimentares comerciais: a proteína hidrolisada vegetal Bio Anastrepha ${ }^{\circledR}$ (Bio Controle, São Paulo, Brasil) e CeraTrap ${ }^{\circledR}$ (Bioibérica S.A, Barcelona, Espanha). Ambos atrativos foram testados nas formas líquida e semissólida. Na forma líquida, a proteína hidrolisada Bio Anastrepha ${ }^{\circledR}$ foi diluída a 5\% e a CeraTrap ${ }^{\circledR}$ sem diluição conforme a indicação dos fabricantes. Para utilização na forma semissólida esses mesmos atrativos foram liofilizados para eliminação da água e concentração dos nutrientes, o atrativo Bio Anastrepha ${ }^{\circledR}$ foi liofilizado sem ser diluído.

Os atrativos na forma líquida foram colocados diretamente em armadilhas do tipo frasco caça-mosca, adaptada a partir de garrafas plásticos transparentes com capacidade para $500 \mathrm{ml}$ contendo três aberturas circulares de $1,5 \mathrm{~cm}$ de diâmetro equidistantes localizadas na parte superior dos recipientes. Onde adicionou-se $325 \mathrm{ml}$ de atrativo líquido por armadilha. Para os atrativos de forma semissólida foram colocados o conteúdo concentrado em um recipiente plástico $(1,5 \times 1,0 \mathrm{~cm})$ que foi suspenso por um arame galvanizado na parte superior e interna das armadilhas (frasco caça-mosca) onde colocouse $325 \mathrm{ml}$ de água destilada.

$\mathrm{O}$ delineamento experimental utilizado foi em blocos casualizados em esquema fatorial $(5 \times 6 \times 2 \times 4)$ sendo: cinco referente a quatro atrativos mais uma testemunha de água destilada, seis períodos de exposição dos atrativos no ambiente telado, dois sexos (insetos fêmeas e machos de C. capitata), todos distribuídos em quatro blocos. Os tratamentos foram dispostos dentro do ambiente telado a uma distância de $4 \mathrm{~m}$ do centro, onde foi colocado um recipiente circular de plástico transparente $(15 \times 30 \mathrm{~cm})$ contendo aberturas circulares de $1,5 \mathrm{~cm}$ de diâmetro, de onde foram liberados 300 adultos recémemergidos na proporção de 1:1 (macho: fêmea) de $C$. capitata. As armadilhas com os atrativos e o recipiente com os insetos foram suspensos a 1,5 $\mathrm{m}$ de altura.

Após cinco dias de exposição dos atrativos em ambiente telado foram realizadas seis liberações dos insetos em períodos intercalados de cinco dias. Realizou-se as contagens após 24 horas da liberação para identificação do número de insetos (fêmeas e machos) capturados em cada armadilha, após essa contagem o conteúdo de cada armadilha foi recolocado na armadilha.

Para determinar a qualidade dos atrativos foram feitas análises iniciais do conteúdo de proteínas dos atrativos líquidos e semissólidos e do $\mathrm{pH}$ apenas nos atrativos líquidos, e mais duas aos 15 e 30 dias após a exposição dos atrativos em ambiente telado. As análises do conteúdo de proteína bruta $(\%)$ dos atrativos foram realizadas no Laboratório de Análise de Alimentos do Departamento de Zootecnia e as avaliações do pH no 
Laboratório de Entomologia, ambos no CCA/UFPB, Areia-PB. Aferiu-se também o volume dos atrativos líquidos e pesaram-se os atrativos semissólidos, antes e após 30 dias de serem expostos no ambiente para verificar a perda por evaporação ao longo do tempo.

Para comprovar a eficiência dos atrativos (atratividade e qualidade dos atrativos), os dados experimentais foram submetidos a análise de variância, e comparados pelo teste de Scott-Knott $(\mathrm{P} \leq 0,01)$. Para avaliar o efeito dos atrativos ao longo dos períodos de exposição os dados foram submetidos a análise de regressão.

\section{Resultados e Discussão}

Durante os seis períodos de exposição dos atrativos, foram capturados um total de 1.051 adultos de $C$. capitata (Tabela 1). Os atrativos diferiram estatisticamente da testemunha quanto ao número de insetos capturados, os atrativos líquidos Bio Anastrepha ${ }^{\circledR}$ e CeraTrap ${ }^{\circledR}$ não diferiram entre si quanto o número de C. capitata capturados, porém diferiram dos demais, no primeiro e segundo período de exposição.

No terceiro e quinto períodos o atrativo líquido CeraTrap ${ }^{\circledR}$ foi o que mais capturou, diferindo estatisticamente dos demais. No quarto período todos os atrativos tiveram comportamento semelhante diferindo apenas da testemunha. No sexto período de exposição o atrativo liquido CeraTrap ${ }^{\circledR}$ e o semissólido Bio Anastrepha ${ }^{\circledR}$ tiveram efeito análogo, porém distinguiram dos demais quanto o número total de moscas capturadas. Os atrativos líquidos Bio Anastrepha ${ }^{\circledR}$ e CeraTrap ${ }^{\circledR}$ foram responsáveis por mais de $60 \%$ do total de moscas capturadas no decorrer dos seis períodos de exposição.

Quando se considerou o total geral de $C$. capitata capturadas nos seis períodos o atrativo líquido CeraTrap ${ }^{\circledR}$, apresentou o maior número de insetos capturados. Segundo um levantamento realizado por Ramos et al. (2011) o atrativo líquido CeraTrap ${ }^{\circledR}$ foi mais eficiente que outros atrativos proteicos no controle de moscas-das-frutas no México, principalmente no controle de Anastrepha spp. Lasa et al. (2013), também constataram a eficiência de CeraTrap ${ }^{\circledR}$ líquida na captura de Anastrepha ludens (Loew) quando comparadas com outros hidrolisados proteicos em diferentes períodos. Utilizando a espécie Anastrepha spp., Raga et al. (2006) compararam a eficiência de atrativos alimentares e confirmaram que o hidrolisado proteico Bio Anastrepha ${ }^{\circledR}$ na forma líquida, foi o mais eficiente na captura, no entanto o conteúdo líquido das armadilhas foi renovado a cada 15 dias. 
Tabela 1. Número médio de adultos de Ceratitis capitata capturados em armadilhas com diferentes atrativos alimentares em diferentes períodos de exposição.

\begin{tabular}{lccccccc}
\hline \multirow{2}{*}{ Tratamentos } & \multicolumn{7}{c}{ Períodos de exposição } \\
\cline { 2 - 8 } & $1^{\mathbf{0}}$ & $2^{\mathbf{o}}$ & $3^{\mathbf{o}}$ & $4^{\mathbf{0}}$ & $5^{\mathbf{0}}$ & $6^{\mathbf{o}}$ & Total \\
\hline Testemunha & $0,38 \mathrm{~b}$ & $0,00 \mathrm{~b}$ & $0,13 \mathrm{c}$ & $0,00 \mathrm{~b}$ & $0,00 \mathrm{c}$ & $0,00 \mathrm{~b}$ & $0,08 \mathrm{~d}$ \\
Bio Anastrepha ${ }^{\circledR} \mathrm{L}$. & $9,75 \mathrm{a}$ & $9,38 \mathrm{a}$ & $6,13 \mathrm{~b}$ & $4,63 \mathrm{a}$ & $2,50 \mathrm{c}$ & $1,63 \mathrm{~b}$ & $5,66 \mathrm{~b}$ \\
Bio Anastrepha ${ }^{\circledR} \mathrm{S}$. & $2,25 \mathrm{~b}$ & $1,63 \mathrm{~b}$ & $4,63 \mathrm{~b}$ & $6,00 \mathrm{a}$ & $5,25 \mathrm{~b}$ & $5,88 \mathrm{a}$ & $4,27 \mathrm{c}$ \\
CeraTrap ${ }^{\circledR} \mathrm{L}$. & $7,88 \mathrm{a}$ & $7,50 \mathrm{a}$ & $8,88 \mathrm{a}$ & $7,50 \mathrm{a}$ & $9,25 \mathrm{a}$ & $8,38 \mathrm{a}$ & $8,22 \mathrm{a}$ \\
CeraTrap ${ }^{\circledR} \mathrm{S}$. & $2,00 \mathrm{~b}$ & $2,75 \mathrm{~b}$ & $4,00 \mathrm{~b}$ & $5,00 \mathrm{a}$ & $4,38 \mathrm{~b}$ & $3,75 \mathrm{~b}$ & $3,64 \mathrm{c}$ \\
\hline
\end{tabular}

Médias seguidas de mesma letra minúscula na coluna não diferem entre si pelo teste de Scott-Knott (P $\leq 0,01)$. L: Líquido, S: Semissólidos.

Ramos et al. (2011), Herrera et al. (2015), Lasa e Cruz (2014), também alcançaram resultados semelhantes ao desta pesquisa, ambos observaram que ao longo de quatro semanas o atrativo CeraTrap ${ }^{\circledR}$, manteve a atratividade e durabilidade, tendo em vista que esse período corresponde apenas ao tempo da pesquisa e não ao período total de validade desse atrativo. O CeraTrap ${ }^{\circledR}$ líquido pode manter suas características viáveis por um período bem mais longo sendo necessário apenas a reposição do conteúdo evaporado (MARÍN, 2010).

O atrativo liquido Bio Anastrepha ${ }^{\circledR}$ manteve a média de captura nos dois primeiros períodos de exposição, contudo a partir do terceiro apresentou um decréscimo na sua atratividade, já o CeraTrap ${ }^{\circledR}$ não oscilou quanto a captura em todos os períodos (Figura 1A, C). Nunes et al. (2013) relataram resultados semelhantes aos do presente estudo, quando avaliaram a eficiência desse atrativo na captura da mosca-das-frutas A. fraterculus (Wiedemann). Quanto aos atrativos na forma semissólida, observaram-se que os dois aumentaram seu potencial de captura a partir do terceiro período de exposição (Figura 1B, D). Resultados semelhantes em que iscas líquidas superaram iscas secas em índice de atratividade foram descritos por Pingel et al. (2006), em monitoramento de moscas-dasfrutas em um cultivo de Sapoti Manilkara achras. Thomas et al. (2008) também verificaram diferenças positivas para as iscas líquidas em relação as iscas secas quando utilizados para captura de A. oblíqua em pomares de manga Mangifera indica.

A manutenção da atratividade da isca líquida CeraTrap ${ }^{\circledR}$ em todos os períodos de exposição, pode estar relacionada ao fato desta não precisar ser diluída, acreditando-se 
que assim não ocorreria uma degradação mais intensa em função das características relacionadas a sua composição. Em sua formulação pode ter um forte conservante que mantem suas características ativas por períodos longos (LASA e CRUZ, 2014), além disso a consistência oleosa pode minimizar os efeitos da degradação provocados pelo ambiente. O atrativo líquido Bio Anastrepha ${ }^{\circledR}$ é usado de forma diluída o que favorece a sua maior degradação sob as condições ambientais. A decomposição e o envelhecimento do atrativo alimentar podem estar diretamente relacionados à eficiência de captura de moscas-das-frutas, variando de acordo com o tipo de atrativo e as influências sofridas quando expostos ao ambiente (RODRIGUES, 2015). Além de ambos apresentarem composição distinta quanto a sua origem, sendo um de origem vegetal e outro animal.

Figura 1. Comportamento dos atrativos alimentares na captura de Ceratitis capitata expostos em diferentes períodos. ${ }^{* *}$ significativo a $(\mathrm{P} \leq 0,01)$. A: Bio Anastrepha ${ }^{\circledR}$ líquida. B: Bio Anastrepha ${ }^{\circledR}$ semissólida. C: CeraTrap ${ }^{\circledR}$ líquida. D: CeraTrap ${ }^{\circledR}$ semissólida
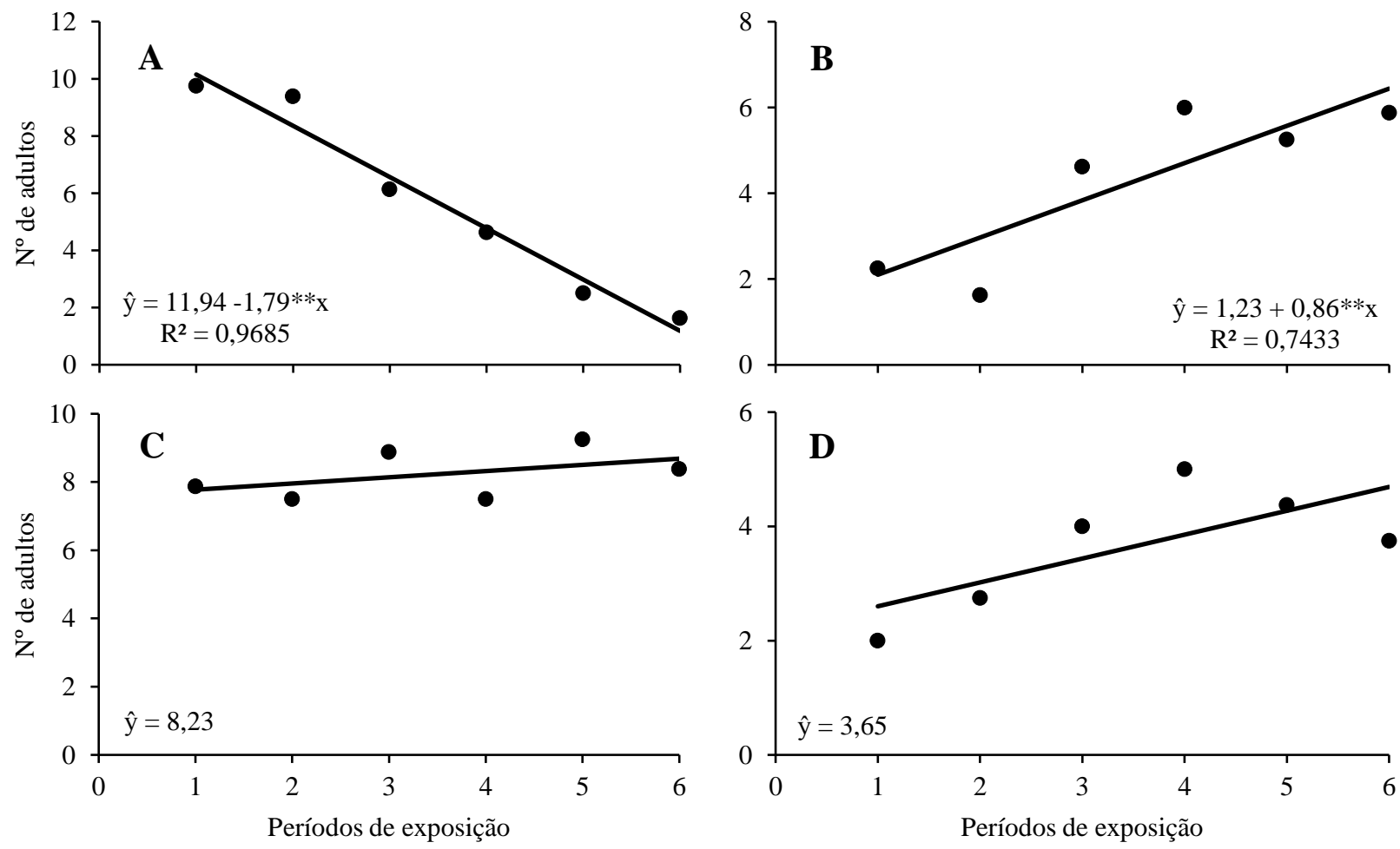

Em ensaios avaliando a eficiência de atrativos a base de proteína hidrolisada na captura de A. suspensa (Loew), Kendra et al. (2005) relacionaram a baixa efetividade desses atrativos a evolução do processo de degradação e liberação de compostos amoniacais de forma mais acelerada. A produção de amônia chega a ser tão intensa que 
pode provocar a repelência dos adultos funcionando assim o atrativo de forma antagônica (NUNES et al., 2013).

Inicialmente os tratamentos na forma semissólida apresentaram menor captura quando comparados aos na forma líquida. Deve-se levar em conta o fato destes apresentarem baixa umidade, característica que pode limitar o alcance dos compostos voláteis dos atrativos alimentares pelos insetos. Segundo Visser (1986), existem fatores que podem determinar a eficiência da captura com atrativos em armadilhas, tais como a maneira que o odor é disperso, a quantidade de odor liberado pela fonte por unidade de tempo, e os mecanismos de orientação do inseto, sendo este último considerado por Rodrigues (2015) o fator mais importante.

Do total de adultos de C. capitata capturados, verificou-se 880 fêmeas $(83,7 \%)$ e 171 insetos machos (16,2\%). Nos tratamentos avaliados exceto a testemunha, observou-se diferenças entre a quantidade de adultos fêmeas e machos capturados. A presença de fêmeas é mais frequente isso porque as fêmeas buscam atrativos proteicos, pois necessitam desses nutrientes para alcançarem a maturidade sexual (KATSOYANNOS et al., 1991) ou seja sua necessidade por alimentos proteicos é maior do que os insetos machos (DÍAZ-FLEISCHER et al., 2009, ARREDONDO et al., 2014).

A avaliação dos tratamentos dentro dos sexos, o número de insetos fêmeas capturados foi maior no atrativo CeraTrap ${ }^{\circledR}$ na forma líquida (Tabela 2). Quanto aos insetos machos não houve diferença significativa entre os atrativos $(\mathrm{F}=1.644 ; p<$ 0,1646), todos apresentaram o comportamento de baixa atratividade quando comparados com o número de fêmeas capturados $(\mathrm{F}=65.953 ; p<0,0001)$. Em levantamentos realizados por Ramos et al. (2011), a relação de insetos fêmeas e machos capturadas pelo o atrativo líquido CeraTrap ${ }^{\circledR}$ demonstrou um índice significativamente mais elevado de fêmeas capturadas das moscas-das-frutas.

O resultado desse estudo é semelhante os obtidos por Hall et al. (2005) e Lasa et al. (2013), que verificaram maior captura de fêmeas em ralação a machos de $C$. capitata e Anastrepha spp. respectivamente usando a isca CeraTrap ${ }^{\circledR}$ líquida. Outros pesquisadores também verificaram a maior captura de insetos fêmeas em relação aos machos utilizando outros hidrolisados proteicos (RAGA et al., 2006; RAGA, 2006; MONTES e RAGA; 2006, ROGRIGUES, 2009; NUNES et al., 2013).

A captura das fêmeas de C. capitata com os atrativos líquidos foi semelhante quando avaliado o número de adultos no geral, do início até o último período de exposição 
em contraste, os atrativos na forma semissólida aumentaram o seu potencial na captura a partir do segundo período de exposição dos atrativos mantendo-o até o último período.

Tabela 2. Número médio de insetos fêmeas e machos de Ceratitis capitata capturados em armadilhas com diferentes atrativos alimentares.

\begin{tabular}{lcc}
\hline \multirow{2}{*}{ Tratamentos } & \multicolumn{2}{c}{ Sexo } \\
\cline { 2 - 3 } & F & M \\
\hline Testemunha & $0,04 \mathrm{dA}$ & $0,13 \mathrm{aA}$ \\
Bio Anastrepha ${ }^{\circledR} \mathrm{L}$. & $9,67 \mathrm{bA}$ & $1,67 \mathrm{aB}$ \\
Bio Anastrepha ${ }^{\circledR} \mathrm{S}$. & $7,08 \mathrm{cA}$ & $1,46 \mathrm{aB}$ \\
CeraTrap $^{\circledR} \mathrm{L}$. & $14,08 \mathrm{aA}$ & $2,38 \mathrm{aB}$ \\
CeraTrap $^{\circledR} \mathrm{S}$. & $5,79 \mathrm{cA}$ & $1,50 \mathrm{aB}$ \\
\hline
\end{tabular}

Médias seguidas de mesma letra, minúscula na coluna e maiúscula na linha não diferem entre si pelo teste de Scott-Knott $(\mathrm{P} \leq 0,01)$. L: Líquido, S: Semissólidos.

O conteúdo de proteína bruta (\%) dos atrativos na forma semissólida não diferiu estatisticamente entre si, quando não expostos e quando expostos, em ambiente telado após 15 dias. Após 30 dias de exposição o atrativo com maior quantidade de proteína bruta $(\%)$ foi o CeraTrap ${ }^{\circledR}$ na forma semissólida. Os atrativos líquidos apresentaram as menores quantidades de proteína bruta. Quando foram avaliados os atrativos nos períodos de exposição constatou-se que foram similares ao conteúdo de proteínas, quando não expostos e quando expostos no ambiente (Figura 2).

O conteúdo de proteína dos atrativos pode está diretamente relacionada com o poder de atratividade das iscas alimentares, em virtude que os adultos de moscas-das-frutas necessitam de alimentos com proteínas em sua composição. Nas fêmeas estas são importantes para que haja o desenvolvimento do ovário, para alcançar sua maturação sexual (DÍAZ-FLEISCHER et al., 2014), para os insetos machos são importantes no desenvolvimento da glândula salivar, para produzir o feromônio sexual (ALUJA, 1989). As proteínas também são importantes para os adultos de moscas-das-frutas porque estão diretamente relacionadas com sua longevidade (DÍAZ-FLEISCHER et al., 2009).

As maiores quantidades de proteínas estão contidas nos atrativos na forma semissólida, no entanto sua eficácia foi inferior na captura de $C$. capitata, sendo necessário pesquisas que viabilizem e ajustem esses atrativos, pois deve-se considerar 
que estes demandam de menos mão-de-obra para sua manutenção e também possuem alto potencial na captura desses tefritídeos.

Figura 2. Conteúdo de proteína bruta dos atrativos expostos em ambiente. A: Bio Anastrepha ${ }^{\circledR}$ líquida. B: Bio Anastrepha ${ }^{\circledR}$ semissólida. C: CeraTrap ${ }^{\circledR}$ líquida. D: CeraTrap ${ }^{\circledR}$ semissólida
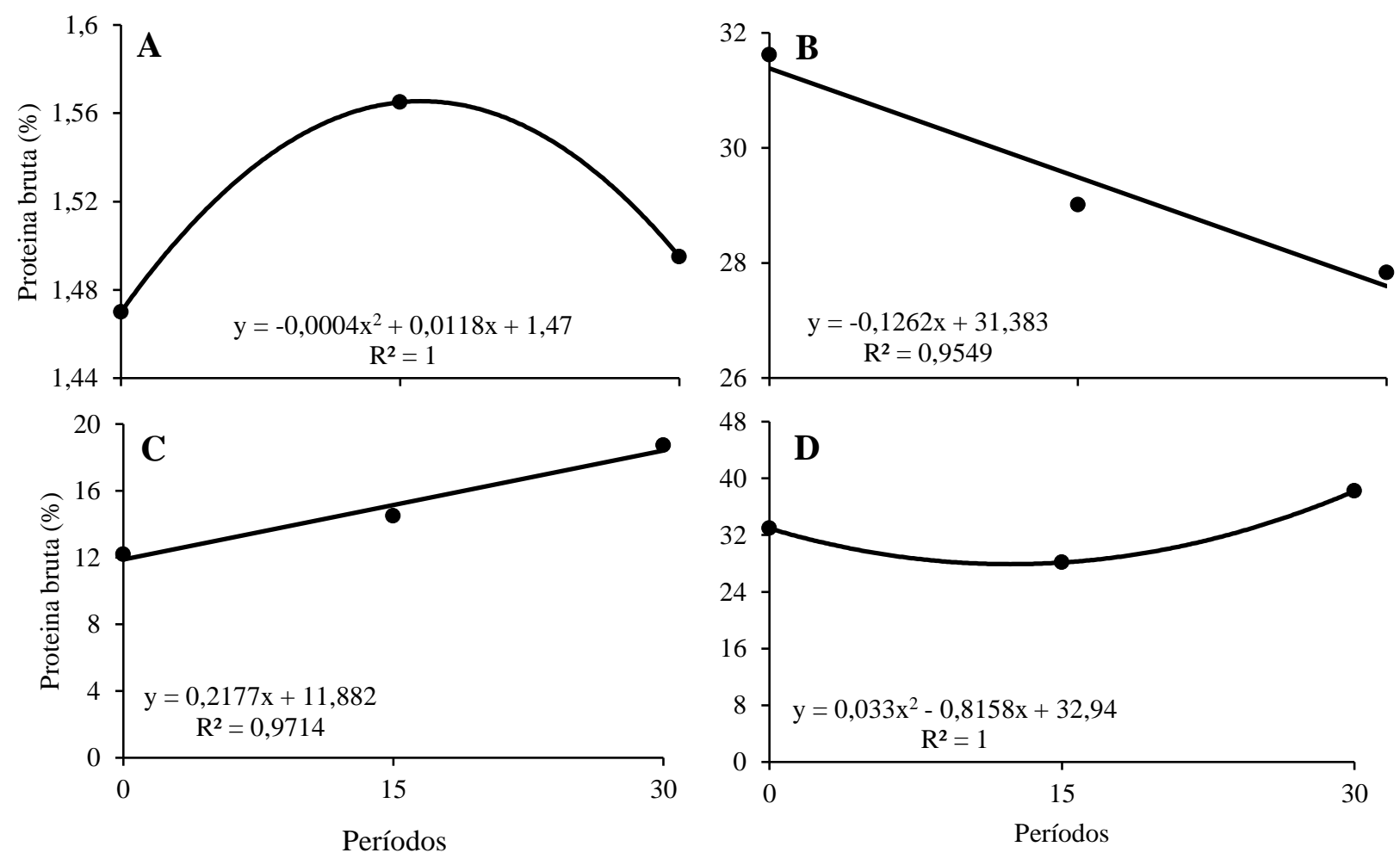

Quando foi mensurado o $\mathrm{pH}$ dos atrativos líquidos verificou-se que existem diferenças significativas quando estes foram ou não expostos ao ambiente. $\mathrm{O}$ Bio Anastrepha ${ }^{\circledR}$ apresenta o pH mais básico que o CeraTrap ${ }^{\circledR}$, em todas as condições de exposição, sendo após 15 dias de exposição o valor mais alto (Tabela 4). Montes e Raga (2006), também observaram resultados semelhantes no $\mathrm{pH}$ do atrativo Bio Anastrepha ${ }^{\circledR}$ quando não exposto e quando exposto ao ambiente por sete dias. O CeraTrap ${ }^{\circledR}$ apresentou o pH mais básico quando não exposto ao ambiente, no entanto a partir de 15 dias de exposição já foi possível observar o pH levemente mais ácido. 
Tabela 4. pH dos atrativos alimentares expostos em diferentes períodos em ambiente telado.

\begin{tabular}{|c|c|c|c|}
\hline \multirow{2}{*}{ Tratamentos } & \multicolumn{3}{|c|}{ Períodos de exposição } \\
\hline & 0 & $15^{\circ}$ dias & $30^{\circ}$ dias \\
\hline Bio Anastrepha ${ }^{\circledR} \mathrm{L}$ & $7,75 \mathrm{aB}$ & $8,57 \mathrm{aA}$ & $7,83 \mathrm{aB}$ \\
\hline CeraTrap ${ }^{\circledR}$ L. & $7,35 \mathrm{bA}$ & $6,80 \mathrm{bB}$ & $6,64 b C$ \\
\hline
\end{tabular}

Médias seguidas de mesma letra, minúscula na coluna e maiúscula na linha não diferem entre si pelo teste de Scott-Knott $(\mathrm{P} \leq 0,01)$. L: Líquido, S: Semissólidos.

$\mathrm{O} \mathrm{pH}$ básico pode influenciar diretamente na eficiência dos atrativos proteicos na captura, monitoramento e controle das moscas-das-frutas em função da liberação de compostos amoniacais, pois estes são os principais voláteis associados a atração de moscas-das-frutas (Montes e Raga 2006). Rodrigues (2015) verificou pH mais básico no atrativo Bio Anastrepha ${ }^{\circledR}$ segundo o autor a manutenção do $\mathrm{pH}$ permitiu um maior período de ação da solução atrativa em campo, na captura de moscas-das-frutas. Raga et al. (2006) também relacionaram a maior captura de adultos de moscas-das-frutas aos atrativos com o pH básico, pois nesse estado há uma maior liberação de voláteis como a amônia, que tendem deixar o pH do atrativo mais básico (Rodrigues 2015). Piñero et al. (2015) relacionaram a baixa atratividade de iscas proteicas a moscas-das-frutas com as baixas taxas de libertação de compostos amoniacais.

Os atrativos líquidos mostraram diferenças em relação a quantidade de líquido evaporado após o período de 30 dias expostos ao ambiente, o atrativo Bio Anastrepha ${ }^{\circledR}$ $(146,7 \mathrm{ml})$ apresentou maior evaporação que o CeraTrap ${ }^{\circledR}(120,0 \mathrm{ml})$. Quanto aos atrativos na forma semissólida não houve perdas no conteúdo e sim um ganho no peso. O Bio Anastrepha ${ }^{\circledR}(1,5 \mathrm{~g})$ apresentou maior ganho que o CeraTrap ${ }^{\circledR}(1,3 \mathrm{~g})$. A menor perda do conteúdo do CeraTrap ${ }^{\circledR}$ pode estar relacionado a maior concentração de seu conteúdo, uma vez que este não é diluído para ser exposto em campo. Já o aumento do peso dos atrativos na forma semissólida pode ser por conta da absorção da umidade, quando expostos no ambiente.

Em ensaios conduzidos por Lasa et al. (2014) o atrativo líquido CeraTrap ${ }^{\circledR}$ teve menos de $50 \%$ de seu conteúdo evaporado durante o período de três meses, quando as armadilhas estavam expostas em campo os autores afirmaram que essa baixa evaporação pode estar relacionada com as baixas temperaturas e a alta umidade observadas no período de exposição dos atrativos em campo. Além disso, a taxa de evaporação também pode depender do diâmetro do orifício das armadilhas. Em estudo relacionado a perda do 
líquido das armadilhas, Pasini et al. (2015) verificaram que armadilhas com aberturas maiores são responsáveis por maiores perdas por evaporação os autores associaram ainda que as armadilhas com menores quantidades de atrativo líquido, são menos efetivas na captura de moscas-das-frutas. Fato esse que pode ajudar a justificar porque nesta pesquisa o atrativo liquido CeraTrap ${ }^{\circledR}$ foi o que mais capturou a referida praga.

\section{Considerações finais}

Os atrativos são mais efetivos na captura de insetos fêmeas da mosca-das-frutas Ceratitis capitata.

O atrativo CeraTrap ${ }^{\circledR}$ na forma líquida é o mais indicado na captura da mosca-dasfrutas Ceratitis capitata, tendo em vista que este apresenta melhor $\mathrm{pH}$, maior conteúdo de proteína bruta e menor taxa de evaporação ao longo do tempo.

\section{Referências}

ALUJA, M.; CABRERA, M.; GUILLEN, J.; CELEDONIO, H.; AYORA, F. Behavior of Anastrepha ludens, A. obliqua and A. serpentina (Diptera: Tephritidae) on a wild mango tree (Mangifera indica) harbouring tree McPhail traps. Insect Sciense and its Application, v. 10, n. 3, p. 309-318, 1989.

ARREDONDO, J.; FLORES, S.; MONTOYA, P.; DÍAZ-FLEISCHER, F. Effect of multiple endogenous biological factors on the response of the tephritids Anastrepha ludens and Anastrepha obliqua to MultiLure traps baited with Bio- Lure or NuLure in mango orchards. Journal of Economic Entomology, v. 107, p. 1022-1031, 2014.

BRITO, C. H. Controle térmico de mosca-das-frutas (Ceratitis capitata) (Wied.) em frutos da cajazeira (Spondias mombin L.). Tese (Doutorado em Agronomia), Centro de Ciências Agrárias da Universidade Federal da Paraíba. Areia-PB, 2007.

DÍAZ-FLEISCHER, F.; ARREDONDO, J.; FLORES, S.; MONTOYA, P.; ALUJA, M. There is nomagic fruit fly trap: multiple biological factors influence the response of adult Anastrepha ludens and Anastrepha obliqua (Diptera: Tephritidae) individuals to Multilure traps baited with BioLure or NuLure. Journal of Economic Entomology, v. 102, p. 86-94, 2009.

DÍAZ-FLEISCHER, F.; PIÑERO, J. C.; SHELLY, T. E. Interactions between tephritid fruit fly physiological state and stimuli from baits and traps: looking for the pied piper of Hamelin to lure pestiferous fruit flies. In: SHELL, N.; EPSKY, N.D.; JANG, E. B.; REYES-FLORES, J.; VARGAS, R. Trapping and the Detection, Control, and Regulation of Tephritid Fruit Flies. Springer, p.145-172, 2014. 
HALL, D. G.; BURNS, R. E.; JENKINS, C. C.; HIBBARD, K. L.; HARRIS, D. L.; SIVINSK, J. M.; NIGG, H. N. Field comparison of chemical attractants and traps for Caribbean fruit fly (Diptera: Tephritidae) in Florida citrus. Journal of Economic Entomology, v. 98, n. 5, p. 1641-1647, 2005.

HERRERA. F.; MIRANDA, E.; GOMEZ, E.; PRESA-PARRA, E.; LASA, R. Comparison of hydrolyzed protein baits and various grape juice products as attractants for Anastrepha fruit flies (Diptera: Tephritidae). Journal of Economic Entomology, v. 109, n. 1, p. 161-166, 2015.

KATSOYANNOS, B. I.; PAPAJ, D. R.; PROKOPY, R. J. Sex differences in movement between natural feeding and mating sites and tradeoffs between food consumption, mating success and predator evasion in Mediterranean fruit flies (Diptera: Tephritidae). Oecologia, v. 86, p. 223-231. 1991.

LASA, R.; CRUZ, A. Efficacy of commercial traps and the lure CeraTrap against Anastrepha obliqua (Diptera: Tephritidae). Florida Entomologist, v. 97, p. 1369-1377, 2014.

LASA, R.; ORTEGA, R.; RULL, J.; Towards development of a mass trapping device for Mexican fruit fly Anastrepha ludens (Diptera: Tephritidae) control. Florida Entomologist, v. 96, n. 3, p. 1135-1142, 2013.

LASA, R.; VELÁZQUEZ, O. E.; ORTEGA, R.; ACOSTA, E. Efficacy of commercial traps and food odor attractants for mass trapping the Mexican fruit fly Anastrepha ludens. Journal of Economic Entomology, v.107, p.198-205, 2014.

LOZANO-TOVAR, M. D.; GARRIDO-JURADO, I.; LAFONT, F.; QUESADAMORAGA, E. Insecticidal activity of a destruxin-containing extract of Metarhizium brunneum against Ceratitis capitata (Diptera: Tephritidae). Journal of Economic Entomology, v. 108, n. 2, p. 462-472, 2015.

MARÍN, G. C. CeraTrap, un Sistema Eficaz y ecológico Pará el control de la mosca de fruta la. Navarro Montes Narro. 2010. Disponível em: <www.navarromontes.com/manual.aspx? man=32>. Acesso em: 26 de Dez de 2017.

MONTES, S. M. N. M; RAGA, A. Eficácia de atrativos para monitoramento de Ceratitis capitata (Diptera: Tephritidae) em pomar de citros. Arquivos do Instituto Biológico, v. 73, n. 3, p. 317-323, 2006.

NASCIMENTO, A. S.; R. S. CARVALHO. Moscas-das-frutas nos Estados Brasileiros. In: ZUCCHI, R. A.; MALAVASI, A. Moscas-das-frutas de importância econômica no Brasil: Conhecimento básico e aplicado. Ribeirão Preto, Holos, p. 235-239, 2000.

NAVARRO-LLOPIS, V.; VACAS, S. Mass trapping for fruit fly control. In: SHELLY, T.; EPSKY, N.; JANG, E. B.; REYES-FLORES, J.; VARGAS, R. Trapping and the detection, control, and regulation of tephritid fruit flies. Springer, p. 513-555, 2014.

NUNES, M. Z.; SANTOS, R. S. S.; BOFF, M. I. C.; ROSA, J. M. Avaliação de atrativos alimentares na captura de Anastrepha fraterculus (Wiedemann, 1830) (Diptera: Tephritidae) em pomar de macieira. Revista de la Facultad de Agronomia, v. 112, n. 2, p. 91-96, 2013. 
PASINI, M. P. B.; LINK, D.; LÚCIO, A, D. C.; FRONZA, D. Hole diameters in pet bottles used for fruit fly capture. Acta Scientiarum, v. 37, n. 2, p.201-209, 2015.

PEREA-CASTELLANOS, C.; PÉREZ-STAPLES, D.; LIEDO, P.; DIAZ-FLEISCHER, F. Escape of Mexican fruit flies from traps baited with CeraTrap and effect of lure feeding on reproduction and survival. Journal of Economic Entomology, v. 108, n. 4, p.1720-1727, 2015.

PIÑERO, J. C.; SOUDER, S. K.; SMITH, T. R.; FOX, A. J.; VARGAS, R. I.

Ammonium acetate enhances the attractiveness of a variety of protein-based baits to female Ceratitis capitata (Diptera: Tephritidae). Journal of Economic Entomology, v. 108, n. 2, p. 694-700, 2015.

PINGEL, R. L.; EPSKY, N. D.; GOENAGA, R. Field trials to attract fruit flies (Diptera: Tephritidae) in commercial sapodilla, mamey sapote and carambola orchards in Puerto Rico. Journal of Agriculture of the University of Puerto Rico, v. 90, p. 109-113, 2006.

RAGA, A. Incidência, monitoramento e controle de moscas-das-frutas na citricultura paulista. Laranja, v. 26, p. 307-322, 2006.

RAGA, A.; MACHADO, R. A.; DINARDO, W.; STRIKIS, P. C. Eficácia de atrativos alimentares na captura de moscas-das-frutas em pomar de citros. Bragantia, v. 65, n. 2, p. 337-345, 2006.

RAGA, A; SOUZA FILHO; M. F.; SATO, M. E.; CERÁVOLO, L. C. Dinâmica populacional de adultos de moscas-das-frutas (Diptera: Tephritidae) em pomar de citros de Presidente Prudente, SP. Arquivos do Instituto Biológico, v. 63, n. 2, p. 23-28, 1996.

RAMOS, M. L. S.; BELLO, A. R; HERNÁNDEZ-PÉREZ, R.; LEAL-GARCIA, D. F. Efec tividad de la estación cebo $\mathrm{ms} 2^{\circledR}$ y atrayente alimenticio CeraTrap ${ }^{\circledR}$ como alternativa en la captura de moscas de la fruta en Veracruz, México. Interciência, v. 37, p. 279-83, 2012.

RAMOS, M. L. S.; HERNAÁNDEZ, R.; CERDÁ, J. M.; NIEVES, F.; TORRES, J. A.; BELLO, A.; FRANCO, D. An environmentally friendly alternative (MS2-CeraTrap) for control of fruit flies in Mexico. Journal of Food, Agriculture and Environment, v. 9, p. 926-927, 2011.

RODRIGUES, M. D. A.; RAGA, A.; MALDONADO JÚNIOR, W.; BARBOSA, J. C. Comparison of food attractants for monitoring fruit fly (Diptera: Tephritidae) in citrus orchards in Brazil. Acta Horticulturae, v. 1065, n. 129, p.1033-1040, 2015.

THOMAS, D. B.; EPSKY, N. D.; SERRA, C.; HALL, D. G.; KENDRA, P. E.; HEATH, R. R. Ammonia formulations and capture of Anastrepha fruit flies (Diptera: Tephritidae). Journal of Entomological Science, v. 43, p. 76-85, 2008.

ZANARDI, O. Z.; NAVA, D. E.; BOTTON, M.; GRÜTZMACHER, A. D.; MACHOTA JR, R.; BISOGNIN, M. Desenvolvimento e reprodução da mosca-domediterrâneo em caquizeiro, macieira, pessegueiro e videira. Pesquisa Agropecuária Brasileira, v. 46, n. 7, p. 682-688, 2011. 
ZUCCHI, R. A. Fruit flies in Brazil: Anastrepha species their host plants and parasitoids. 2008. Available in: <www.lea.esalq.usp.br/anastrepha〉. Acesso em: 15 de Dez. de 2017. 